\title{
Introdução
}

\section{A sociologia e suas Interfaces com contextos local, nacional e global}

\begin{abstract}
Carlos Benedito Martins*
Resumo: $\mathrm{O}$ artigo destaca que, ao lado da presença da sociologia no interior de diversas sociedades nacionais, nas últimas décadas ocorreu a constituição de um espaço transnacional de produção da sociologia. O surgimento deste espaço transnacional de sociologia apresenta uma estrutura de poder assimétrica, em função da distribuição desigual de recursos materiais e simbólicos entre os diferentes países. Salienta a relevância da utilização de uma sociologia reflexiva, que preconiza uma constante reflexão sobre as condições históricas, sociais, institucionais e acadêmicas que se encontram presentes na produção do conhecimento sociológico, capaz de elucidar as complexas relações existentes entre este novo espaço transnacional da disciplina e as diversas sociologias nacionais.
\end{abstract}

Palavras-chave: Sociologias nacionais. Globalização da sociologia. Estrutura de poder no espaço transnacional da sociologia. Diversificação da produção internacional da sociologia.

\section{Sociology and its Interfaces \\ with local, national and global contexts}

The article emphasizes that, in addition to the presence of sociology within different national societies, in the last decades the creation of a transnational space for the production of sociology took place. The emergence of this transnational space of sociology presents an asymmetric power structure, due to the unequal distribution of material and symbolic resources between countries. It underlines the importance of using a reflexive sociology, which calls for a constant reflection on the historical, social, institutional and academic conditions that are present in the production of sociological knowledge, able to elucidate the complex relationship between this new transnational space of discipline and the various national sociologies.

Keywords: National Sociologies. Globalization of sociology. Power structure in the transnational space of sociology. Diversification of the international production of sociology.

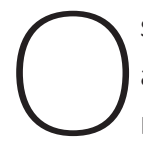
s textos publicados neste número da Revista Sociedade e Estados reúnem as conferências que foram realizadas pelos convidados internacionais durante o XVIII Congresso Brasileiro de Sociologia, que ocorreu na cidade de Brasília no mês de julho de 2017, e teve como temática principal a indagação: "Que

\author{
* Carlos Benedito \\ Martins é \\ professor titular \\ do Departamento \\ de Sociologia da \\ Universidade de \\ Brasília, Brasília, \\ Distrito Federal, \\ Brasil. Foi visiting \\ scholar em diversas \\ instituições, \\ dentre as quais \\ a Universidade \\ de Oxford, a \\ Universidade \\ de Colúmbia, \\ a Universidade \\ Livre de Berlim, a \\ Universidade Hong \\ Kong, a Universidade \\ Nacional de \\ Singapura, a École \\ des Hautes Études \\ em Sciences Sociales. \\ <carlosb@unb.br>.
}


sociologias fazemos? Interfaces com contextos local, nacional e global". O tema escolhido - sem abrir mão de questões candentes da sociedade contemporânea e especialmente do contexto nacional - visou incentivar a reflexão a propósito da multiplicidade de configurações temáticas e teóricas que perpassam a produção sociológica contemporânea tanto no contexto internacional quanto nacional. A leitura das diferentes conferências evidenciam a pluralidade de questões exploradas e também a diversidade de abordagens explicativas mobilizadas pelos conferencistas provenientes de diferentes regiões, tais como África do Sul, Austrália, França, Alemanha, Inglaterra, Síria, Estados Unidos, Singapura etc. O congresso contou ainda com a participação de acadêmicos provenientes de outras partes do mundo, que se envolveram em diversas atividades nos grupos de trabalho, nos fóruns e/ou nas mesas redondas. A presença destes participantes internacionais propiciou informações relevantes sobre os contextos históricos, sociais, institucionais e acadêmicos nos quais encontram ancorados suas produções acadêmicas.

Simultaneamente, a presença no XVIII Congresso da Sociedade Brasileira de Sociologia (SBS) de destacados sociólogos seniors nacionais e também a participação de uma nova e promissora geração de sociólogos que atuam profissionalmente em diversas regiões do país, inseridos em 40 grupos de trabalho, 45 mesas redondas, em uma dezena de minicursos, forneceram informações pertinentes sobre a variedade de temas de pesquisa que vêm sendo explorados atualmente pela sociologia realizada no Brasil. Ao mesmo tempo, esta participação da comunidade sociológica nacional colocou em evidência o papel público que ela vem exercendo diante dos desafios sociais, políticos, econômicos e culturais do país.

De certa forma, a temática do congresso inspirou-se na esteira de uma sociologia reflexiva, ou seja, de uma sociologia da sociologia que preconiza a pertinência da prática de uma constante reflexão sobre as condições históricas, sociais, institucionais e acadêmicas presentes na produção do conhecimento sociológico. Para a diretoria da Sociedade Brasileira de Sociologia (SBS 2015-2017), esta postura reflexiva constitui uma condição essencial para preservar a posição pertinente da sociologia na esfera cognitiva da sociedade brasileira e sua inserção no cenário internacional, diante das complexas transformações que estão ocorrendo no âmbito da própria disciplina.

As diversas sociologias nacionais encontram-se diante de profundas transformações culturais, socioeconômicas e políticas que têm perpassado em ritmos diferentes as sociedades contemporâneas, cujas mudanças têm reverberado direta e/ou indiretamente nas abordagens teóricas e nos temas de investigação que vêm sendo explorados pelos pesquisadores no contexto atual da produção do conhecimento 
sociológico. Ao lado da presença da sociologia no interior de diversas sociedades nacionais, é plausível identificar a constituição de um espaço transnacional de produção da sociologia, que se manifesta empiricamente através de um intenso fluxo de mobilidade de pesquisadores e de ideias que se estendem além das fronteiras nacionais, pela intensa circulação mundial de periódicos internacionais, pela proliferação de simpósios internacionais que contam com a participação de sociólogos de diferentes partes do mundo, bem como pela formação de redes internacionais de pesquisa. A formação deste espaço transnacional da sociologia e a progressiva constituição de uma "comunidade sociológica global" - segundo a expressão de Piotr Sztompka (2010) - têm impulsionado diversas sociologias nacionais, situadas tanto em centros hegemônicos como em polos emergentes, a analisar sociologicamente a produção sociológica que vem sendo praticada em seus respectivos países.

Nos últimos anos tem surgido uma leva de trabalhos reflexivos - que se aproximam de uma sociologia da sociologia - sobre a configuração de um espaço internacional da produção sociológica e sua interseção com as sociologias nacionais. Nesta direção, vale mencionar brevemente, entre outros, o trabalho de Gurminder Bhambra (2014) Connected sociologies, a edição coordenada por Didier Demazière (2016) Les sociologies françaises: héritages et perpectives 1960-2010, o livro coletivo editado por Sujata Patel (2011) denominado Doing sociology in India: genealogies, locations and practices, o livro organizado por Craig Calhoun(2007) Sociology in America: an introduction, o relatório produzido por Michel Wieviorka e Jacques Moret (2017) Les sciences humaines et sociales françaises à l'échelle de l'Europe et du monde etc. Deve-se também destacar a existência de um volume expressivo de artigos reflexivos sobre a emergência deste espaço internacional da sociologia e/ou voltados para o processo de inserção das diversas sociologias nacionais no interior deste espaço, publicados em revistas tais como Current Sociology e Internacional Sociology, dentre outros periódicos internacionais e nacionais.

O ritmo veloz e a amplitude de mudanças sociais, políticas e culturais, que estão ocorrendo em diversas sociedades contemporâneas, vêm apresentando desafios para o próprio universo empírico da sociologia, ou seja, o terreno de análise no qual esta disciplina concentrou suas análises ao longo do tempo. A este propósito, deve-se assinalar que durante um extenso período, o terreno predominante de investigação da sociologia tem sido a análise das diversas sociedades nacionais, seja no continente europeu, seja na América do Norte, Ásia, África, seja na América Latina. A própria noção de sociedade, particularmente entre os pensadores do século XIX, estava, de certa forma, relacionada à existência do Estado-nação. A este propósito, vale assinalar que a institucionalização da sociologia, no final do século XIX, ocorreu num momento em que o princípio de nacionalidade se afirmava com toda 
a força. Enquanto tendência predominante no interior da sociologia, as sociedades nacionais têm sido analisadas como unidades autônomas, fechadas em si mesmas, separadas umas das outras pela delimitação de seus territórios. Tanto assim que se fala de uma sociologia francesa, alemã, norte-americana, brasileira etc. O ponto focal das investigações centradas nas sociedades nacionais visou compreender o interior de cada uma delas, procurando captar sua estrutura social, a articulação de suas instituições, seus padrões de desigualdade e modos de conflito e o processo de mudanças sociais (Heilbron, 2015; Levine, 1985).

Certamente, as diversas sociedades nacionais continuam a existir, manifestando-se por meio da defesa de seus territórios, de suas tradições culturais, acadêmicas etc. Nas sociedades nacionais, tendem a perdurar sentimentos nacionalistas, regionalistas, reivindicações de pertencimentos étnicos etc. (Turner, 2007). No entanto, as profundas transformações que estão ocorrendo em escala global nas sociedades contemporâneas tendem a indicar que a existência das sociedades nacionais não esgota a complexidade da realidade contemporânea com a qual a sociologia deve lidar. Gradativamente, nas últimas décadas do século XX, surgiu um conjunto de fenômenos econômicos, políticos, culturais, militares, acadêmicos, entre outros, que operam em uma escala que transcende as fronteiras das diversas sociedades nacionais. Neste contexto, ocorre o incremento de conexões econômicas, políticas, culturais, acadêmicas etc., entre diferentes localidades, países, empresas, finanças, indivíduos, movimentos sociais etc. Nesse sentido, forma-se uma densa rede de relações sociais, econômicas, políticas, culturais e acadêmicas que operam num nível supranacional. A presença destes fenômenos - ora analisados como expressão da globalização e/ou mundialização - alargou de forma considerável o terreno empírico e o âmbito da análise da sociologia, até então concentrados predominantemente nas sociedades nacionais, conforme assinalado anteriormente, estendendo-os para um plano transnacional e/ou global (Hsu, 2010; Turner \& Khondker, 2010; Touraine, 2004; Robertson, 2000). Ao mesmo tempo, a presença destes fenômenos transnacionais tende a demandar novos instrumentos de análise da sociologia, visando captar as complexas relações entre as articulações dos níveis local, regional, nacional e global que se encontram entrelaçados de modo singular em várias sociedades contemporâneas (Delanty, 2009; Beck, 2005; 2006).

Uma constelação de fenômenos ocorridos em distintos planos da sociedade contemporânea se entrelaçou de um modo tal que propiciou a constituição progressiva de um espaço transnacional da sociologia. Diante desta nova situação, as relações acadêmicas entre as diversas sociologias nacionais passaram a ocupar outro patamar, quando comparadas a épocas anteriores. Nesse sentido vale destacar o papel desempenhado pela Internacional Sociological Association (ISA), que ampliou o re- 
crutamento de participantes no seu interior. Ao seguir o modelo de sistematização da Organização das Nações Unidas (ONU), de privilegiar as representações nacionais, a ISA - a exemplo de outras associações cientificas internacionais - foi constituída a partir de um pequeno número de associações nacionais que estava concentrado em alguns países europeus e na América do Norte. A partir do final dos anos 1960, a ISA e outras associações permitiram a entrada e a participação de indivíduos em suas atividades, aumentando seu escopo de recrutamento. Com o processo de descolonização que ocorreu nesta época, as nações pós-coloniais e seus pesquisadores também passaram a integrar a ISA. Ao mesmo tempo, ocorreu a entrada de países comunistas do leste europeu no seu interior. Ou seja, a partir dos anos 1970, verificou-se uma ampliação significativa da base geográfica da entidade que, pouco a pouco, teria repercussões nas discussões teóricas e metodológicas promovidas em seu interior (Heilbron, 2014; Heilbron et alii, 2009; Platt, 1998).

Na dinâmica do processo de formação de um espaço transnacional da sociologia, vale destacar o surgimento de novos centros econômicos e acadêmicos dinâmicos na Ásia e em outras regiões do hemisfério sul e o desenvolvimento de novas tecnologias de comunicação, que impulsionaram o incremento do intercâmbio de uma diversidade de orientações teóricas e metodológicas da sociologia provenientes de diversos países. Também contribuíram para a formação deste novo espaço a forte expansão do ensino superior pelo mundo, o incremento da mobilidade acadêmica internacional, a intensificação de debates sobre a disciplina - que atravessam as fronteiras nacionais em ritmo veloz -, a implantação de políticas de ciência e tecnologia empreendidas por diversos países com posições emergentes no universo acadêmico, visando alavancar suas respectivas comunidades de pesquisadores. Algumas análises têm destacado que, no contexto da globalização que perpassa as sociedades contemporâneas, aflorou um ethos cultural que incentiva e valoriza os indivíduos a se movimentarem avidamente em suas vidas privadas e profissionais, incentivando-os a novas e desafiadoras experiências em seus distintos campos de atuação, fenômeno este que estaria subjacente ao incremento da mobilidade acadêmica internacional (Elliot \& Lemert, 2006; Ray, 2007; Elliot \& Urry, 2010). Ao mesmo tempo, deve-se destacar que os governos de vários países têm criado políticas específicas para intensificar a circulação internacional de seus professores, uma vez que a internacionalização do corpo docente passou a ser considerado um aspecto positivo nas avaliações dos rankings mundiais sobre as universidades. O resultado tem sido o incremento das relações acadêmicas entre sociólogos de diferentes países (Cantwell, 2011; Maringe \& Foskett, 2010).

O documento World social science report (Unesco, 2010) traz informações relevantes sobre a constituição do espaço mundial da sociologia. Este trabalho - que con- 
tou com a participação de cientistas sociais destacados, como Craig Calhoun, Saskia Sassen, Peter Wagner e Syed Alatas, dentre outros - indica que as ciências sociais nos dias atuais, ao contrário de seu início, estão presentes em todas as regiões do mundo nas quais existem sistemas de ensino superior. Neste processo de ampliação mundial, formaram-se associações nacionais de sociologia em uma parte expressiva de países e também organismos regionais, visando estimular as ciências sociais que ocupam posições periféricas neste espaço transnacional, como o Arab Council for the Social Sciences (ACSS), a Association of Asian Social Science Research Councils (Aassrec), o Council for the Development of Social Science Research in Africa (Codesria) e o Latin America Council of Social Sciences (Clacso). Simultaneamente, passou a ocorrer um maior afluxo de sociólogos em congressos internacionais, como os da ISA, e também em encontros temáticos, como os da Latin American Studies Association (Lasa), atualmente uma das maiores associações científicas transnacionais do mundo, composta por mais de 12 mil sócios, bem como de instituições provenientes de uma multiplicidade de países dedicadas ao estudo da América Latina. Cada vez mais se observa a formação de redes de investigações integradas por pesquisadores oriundos de diferentes países, que trabalham conjuntamente, por um determinado período, em um mesmo objeto, compartilhando fundamentos teóricos e procedimentos metodológicos que tendem a extravasar suas tradições culturais e acadêmicas nacionais. Nesta direção, compartilham ideias comuns, tendem a se reportar às mesmas obras, consultam revistas científicas similares, de tal forma que as diversas sociologias nacionais vêm ultrapassando as fronteiras e atuando, progressivamente, em outro patamar, numa prática já rotineira no contexto da global sociological community, segundo expressão de Piotr Sztompka (2010).

No entanto, o surgimento deste espaço transnacional de sociologia apresenta uma estrutura de poder assimétrica, em função da distribuição desigual de recursos materiais e simbólicos entre os diferentes países. A disparidade decorre das diferentes condições de infraestrutura acadêmica disponíveis em seus países, vale dizer, da qualidade e reputação acadêmica de suas universidades, da capacidade instalada de investigação científica, disponibilidade de financiamento material, recursos humanos para o desenvolvimento de pesquisas e também do reconhecimento social e simbólico dos pesquisadores. Nesse sentido, ocorre uma nítida dominação da produção do conhecimento, de autores, das editoras e das revistas internacionais localizadas em determinados países do ocidente (Gareau, 1988).

Ao tomar como referência duas bases de dados - a Ulrich e a Thomson -, o trabalho World social science report mostra que existe densa concentração da publicação de artigos em revistas internacionais na área da sociologia na Europa e América do Norte. Essas duas regiões concentram aproximadamente $90 \%$ da produção mundial 
na área. A base de dados da Ulrich abrange um número maior de revistas internacionais. Do total de 6.640 revistas, eles selecionaram 3.046, cujos artigos passam pela revisão de pares. Nesta base de dados, tem-se a seguinte distribuição em termos de participação mundial na publicação de artigos: Europa 44\%, América do Norte 37\%, Ásia 9\%, América Latina 5\%, Oceania 4\%, África 2,2\%, Commonwealth e Estados Independentes 0,6\%. Quando se utiliza a base de dados da Thomson, que trabalha com um número menor de revistas internacionais, a disparidade nos índices se acentua. Nesta base de dados, a Europa responde por 46,1\%; América do Norte 46,5\%, Ásia 3,7\%, América Latina 1,3\%, Oceania 1,9\%, África 0,4\%\%, Commonwealth e Estados Independentes 0,1\%.

Os dados mostram o domínio da língua inglesa na circulação das publicações. A base de dados da Ulrich aponta que $85 \%$ dos artigos são publicados em inglês, $6 \%$ em francês, 5\% em alemão, 4\% em espanhol, 1,7\% em português e o restante em outras línguas. No entanto, quando se utiliza a base de dados da Thomson, verifica-se um crescimento da língua inglesa, com 94,45\%; seguindo o alemão, com 2,14\%; o francês, com 1,25\%; o espanhol, com 0,40\%; o português, com 0,08\% etc. As traduções de trabalhos evidenciam também uma forte desigualdade entre as regiões. Predomina a tradução de livros publicados em inglês para as línguas vernáculas dos diferentes países. No entanto, poucos trabalhos relevantes escritos em outras línguas são traduzidos para o inglês. Isso demonstra que a formação de um espaço transnacional da sociologia tem reproduzido a dominação simbólica e material principalmente da América do Norte e de alguns países da Europa. A globalização da vida acadêmica e das ciências sociais sob a hegemonia linguística e científica anglo-saxã tem, em certa medida, conduzido ao enfraquecimento da visibilidade no contexto internacional da sociologia de países que desempenharam papel relevante no processo de emergência desta disciplina (Wieviorka \& Moret, 2017).

No entanto, vários países que ocupam posições emergentes no espaço transnacional das ciências sociais desenvolveram políticas científicas e tecnológicas - por meio de suas agências de financiamento - que tornaram algumas de suas universidades atores estratégicos no processo de institucionalização das ciências sociais. Nesses países, ocorreu a expansão dos cursos de pós-graduação em várias áreas das ciências sociais e particularmente na sociologia. Ao mesmo tempo, criou-se um conjunto de agências regionais que têm desempenhado um papel relevante no processo de desenvolvimento das ciências sociais na América Latina, tais como o Conselho Latino-Americano de Ciências Sociais (Clacso), a Faculdade Latino-Americana de Ciências Sociais (Flacso) (Vessuri, 2015; Vesuri \& Lopez, 2010). O conjunto destas iniciativas situa as ciências sociais destas regiões num nível distinto de qualidade 
acadêmica, quando comparado com décadas anteriores. Indicam também a constituição de um contingente de pesquisadores qualificados, que não apenas estão cada vez mais inseridos no espaço internacional da sociologia, mas que reivindicam uma posição de destaque no seu interior.

Tudo leva a crer que, ao reverberar na sociologia contemporânea o processo de globalização compreendido como fenômeno multidimensional - ou seja, que abarca as dimensões econômicas, políticas e culturais (Turner \& Khondker, 2010) - está se desafiando determinados fundamentos de seu arcabouço explicativo. A gradativa constituição deste espaço transnacional e/ou global no qual a sociologia passou a atuar, concomitantemente às sociologias nacionais, propiciou o aparecimento e a disseminação em vários países de novas abordagens teóricas/explicativas. Numerosos trabalhos ressaltam que determinados conceitos sociológicos usados de forma recorrente - e até então considerados incontroversos - têm se mostrado problemáticos quando são utilizados em contextos não ocidentais. Portanto, as complexas transformações sociais, políticas, culturais e acadêmicas que atravessam diversas sociedades contemporâneas, tal como o impacto destas mudanças no interior da estrutura cognitiva da disciplina, têm instigado diversas sociologias nacionais a incluir em suas pautas de trabalho a necessidade de uma reflexão crítica sobre sua produção e os alicerces cognitivos que as sustentam (Knöbl, 2015; Alatas, 2006; Keim, 2011; 2016; Connell; 2007; 2010).

Cada vez mais se observa nos dias correntes uma forte pressão por parte de agências federais e estaduais de financiamento de ciência e tecnologia para promover a internacionalização da atividade científica produzida no país, cujo discurso reverbera no cotidiano das universidades e nos programas de pós-graduação em praticamente todas as áreas de conhecimento e, certamente no âmbito da sociologia.

Diante desta pressão e também em face das transformações que estão ocorrendo no espaço transnacional da sociologia, que mantém intricadas relações com as diversas sociologias nacionais, o enfrentamento da complexa questão da internacionalização da sociologia realizada no Brasil implica um duplo e simultâneo trabalho de reflexão, ou seja, desenvolver um adequado conhecimento sobre a constituição do espaço transnacional da sociologia, suas regras de funcionamento e estruturas de poder existentes em seu interior e, ao mesmo tempo, desenvolver um olhar crítico sobre a sociologia que vem sendo efetivada atualmente no Brasil. A questão que se coloca é: quais os desafios institucionais que a sociologia que se realiza no país tem diante de si, caso deseje, inserir-se de forma relevante neste novo espaço transnacional da disciplina e como enfrentar a questão do financiamento à pesquisa inerida em redes internacionais. 
O conjunto de trabalhos que integram esta edição da Revista Sociedade e Estado insere-se na dinâmica deste processo de autoanálise, ou seja, da pratica de uma sociologia reflexiva. Neste sentido, os trabalhos apresentados nesta edição e outros que vêm sendo realizados, tal como a edição do livro Sociologia brasileira hoje, organizado por Miceli e Martins (2017), se situam numa agenda de trabalho voltada para refletir de forma recorrente sobre os recortes temáticos, as referências teóricas e metodológicas utilizadas, levando em consideração a existência do espaço transnacional da disciplina, diante do qual cumpre indagar as possibilidades e estratégias possíveis da sociologia realizada no Brasil de ocupar uma posição de destaque neste espaço transnacional.

\section{Referências}

ALATAS, Syed. The autonomus, the universal and the future of sociology. Current Sociology, v. 54, n. 1, p. 7-23, 2006.

ARJOMAND, Said. International sociology into the new millennium: the global sociological community and the challenge to the periphery. International Sociology, $\mathrm{v}$. 15 , n. 1, p. 5-10, 2000.

BECK, Ulrich. Cosmopolitan vision. Cambridge (UK): Polity Press, 2006.

How not to be a museum piece. The Bristish Journal of Sociology, v. 56, n.

3, 2005.

BHAMBRA, Gurminder. Connected sociologies. London: Bloombury, 2014.

CALHOUN, Craig. The sociology in America: a history. Chicago (IL): The Chicago University Press, 2007.

CANTWELL. Brendan. Transnational mobility and international academic employment: gatekeeping in an academic competition arena. Minerva, v. 49, n. 4, p. 425445, 2011.

CONNELL, Raewyn. Learning from each other: sociology on a world scale. In: PATEL, Sujata (Org.). The ISA handbook of diverse sociological traditions. London: Sage, 2010.

Southern theory. The global dynamics of knowledge in social science. Sydney: Allen \&Unwi, 2007.

DELANTY, Gerard. The cosmopolitan imagination: the renewal of critical social theory. Cambridge (UK): Cambridge University Press, 2009. 
DEMAZIÈRE, Didier et alii. Les sociologies françaises: héritages et perspectives (1960-2010). Rennes: Presses Universitaires de Rennes, 2015.

ELLIOT, Anthony; URRY, John. Mobile lives. Oxford (UK): Routledge, 2010.

ELLIOT, Anthony; LEMERT, Charles. The new individualism: the emotional costs of globalization. London: Routledge, 2006.

GAREAU, Frederick. Another type of third dependency: the social sciences. International Sociology, v. 3, n. 2, p. 171-178, 1988.

HEILBRON, Johan. French sociology. Ithaca (NY): Cornnell University Press, 2015.

- The social sciences as an emerged global field. Currenv Sociology, v. 62, n. 5, p. 685-703. 2014.

HEILBRON, Johan et alii. Internationalisation des sciences sociales: les leçons d'une histoire transnationale. In: SAPIRO, Gisèle (Org.). L'espace intellectuel en Europe: de la formation des Étas-nations à la mondialisation: XIX-XX siècles. Paris: La Découverte, 2009.

HSU, Eric. Social theory and globalization. In: ELLIOT, Anthony (Org.). The Routledge companion to social theory. London: Routledge, 2010.

KEIM, Wiebke (Org.). Global knowledge production in the social sciences. London: Routledge, 2016.

. Counterhegemonic currents and internationalization of sociology. International Sociology, v. 26, n. 1, p. 123-145, 2011.

KNÖBL, Wolfgang. Reconfigurações da teoria social após a hegemonia ocidental. Revista de Ciências Sociais, v. 30, n. 87, p. 5-17, 2015.

LEVINE, Donald. Visions of the sociological traditions. Chicago (IL): The University of Chicago Press, 1985.

MARINGE, Felix; FOSKETT, Nick (Orgs.). Globalization and internationalization in higher education: theoretical, strategic and management perspectives. London: Continuum Iternational Publishers, 2010.

MICELI, Sergio; MARTINS, Carlos Benedito. Sociologia brasileira hoje. São Paulo: Ateliê Editorial, 2017.

PATEL, Sujata. Doing sociology in India: genealogies, locations and practices. Oxford (UK): Oxford University Press, 2011. 
PLATT, Jennifer. History of the (ISA) International Sociological Association. Montréal: Université de Québec, 1998.

RAY, Larry. Globalization and evereday. London: Routledge, 2007.

ROBERTSON, Roland. Globalization: social theory and global culture. London: Sage Publication, 2000.

SZTOMPKA, Piotr. One sociology or many? In: PATEL, Sujata (Org.). The ISA handboook of diverse sociological traditions. London: Sage, 2010.

TOURAINE, Alain. Un nouveau paradigme pour comprendre le monde d'aujourd'hui. Paris: Fayard, 2004.

TURNER, Bryan. The enclave society: towards a sociology of immobility. European Journal of Social Theory, v. 10, n.2, p. 287-303, 2007.

TURNER, Bryan; KHONDKER, Habibul. Globalization: East and West. London: Sage, 2010.

UNITED NATIONS EDUCATIONAL, SCIENTIFIC AND CULTURAL ORGANIZATION (UNESCO). World social science report. Knowledge divides. Paris: Unesco, 2010.

VESSURI. Hebe. Global social science discourse: a southern perspective on the world. Current Sociology, v. 63, n. 2, p. 297-313, 2015.

VESSURI. Hebe; LOPEZ, Maria. Institutional aspects of the social sciences in Latin America. World social science report. Paris: International Science Council Unesco Publishing, 2010.

WIEVIORKA, Michel; MORET, Jacques. Les sciences humanines et sociales françaises à l'échelle de l'Europe et du monde. Paris: Éditions de la Maison des Sciences de I'Homme, 2017. 